\title{
A Prospective Study on Monitoring and Evaluation of Adverse Drug Reaction in A Tertiary Care Hospital
}

\author{
Yadhukrishnan $\mathrm{PU}^{1 *}$, Nolin Johncy ${ }^{2}$, Christan $\mathrm{M}^{3}$, Neena Priyamalar $\mathrm{EM}^{3}$, Mohamed Thayub $\mathrm{S}^{4}$, \\ Daniel Sundar Singh $\mathrm{S}^{3}$ \\ ${ }^{T}$ Department of Clinical Pharmacy, Indiana Hospital and Heart Institute, Mangalore, Karnataka India \\ ${ }^{2}$ Department of Pharmacy Practice, Srinivas College of Pharmacy, Mangalore, Karnataka India \\ ${ }^{3}$ Department of Pharmacy Practice, C.L.Baid Metha College of Pharmacy, Chennai, Tamil Nadu India \\ ${ }^{4}$ Department of Clinical Pharmacy, Frontier Lifeline Hospital, Chennai, Tamil Nadu India
}

\section{*Corresponding Author}

Yadhukrishnan P U

\author{
Article History \\ Received: 26.01 .2020 \\ Accepted: 05.03 .2020 \\ Published: 08.03 .2020
}

\begin{abstract}
Background: An Adverse drug reaction (ADR) is an injury caused by taking medication. ADRs are major threat to patient therapy as it results in significant morbidity, mortality and health care costs. Objective: To monitor and evaluate the ADRs in a tertiary care hospital. Methods: This observational and cross-sectional study was conducted for 10 months from January 2019 to October 2019 in an inpatient setting of a tertiary care hospital in Mangalore using ADR reporting form. Patients of all age groups, either sex and only inpatients were included. Results: ADR's caused by Intravenous route were the highest occurring comprising of $60.46 \%$ followed by $29.53 \%$ causing by oral route of administration. Among the organ systems skin membrane were mostly affected which is of $60.46 \%$ and $13.95 \%$ of ADR's were present as CNS and neurological disorders. Drug categories suspected to cause ADRs were related to antibiotics (39.53\%), G1 drugs (16.27\%), and the remaining classes of drugs like anti-diabetics, anti-coagulants etc.11 (26\%) ADR's were found to be mild, 25 (58\%) were moderate and 7 (16\%) of ADR's were found to be severe according to severity assessment. Conclusion: Adverse drug reaction is a significant limitation to the success of therapeutics and to deal with this problem pharmacovigilance program is initiated to emphasise the importance of monitoring and reporting of ADRs to ensure the safety use of drugs. However the quality and quantity of the ADR reports can be improvised only through educational programs that guide the healthcare providers in recognising and reporting the ADRs efficiently.
\end{abstract}

Keywords: Adverse drug reaction, Pharmacovigilance, Hartwig and Siegal scale.

\section{INTRODUCTION}

Drugs are substances that are used for the purpose of diagnosis prevention and treatment of various diseases. But sometimes the drugs tend to exhibit some undesired harmful response, such response are seen even at therapeutic doses and it varies from one person to another [1]. The World Health Organization (WHO) defines an adverse drug reaction (ADR) as "one which is noxious and unintended, and which occurs in doses normally used in human for prophylaxis, diagnosis, or therapy of disease or for the modification of physiological functions [2]. The ADRs are a serious threat to the therapy and health care provided to the patient. It is the leading cause of mortality and morbidity. The criteria for serious adverse drug reactions (serious ADRs) have been specified by the WHO and include any untoward medical occurrence at any dose that results in death, life-threatening, requires or prolongs hospitalization, or results in persistent or significant disability or incapacity. The incidence of ADR also increases the healthcare burden and costs of the patient. Thus monitoring and evaluation of such ADRs are very important in order to prevent similar ADRs in the future and also to develop a management plan. The practice of monitoring, detecting and handling of ADRs is called as Pharmacovigilance. Pharmacovigilance (PV) is defined as the science and activities relating to the detection, assessment, understanding and prevention of adverse effects or any other drug-related problem. The quality and quantity

Copyright @ 2020: This is an open-access article distributed under the terms of the Creative Commons Attribution license which permits unrestricted use, distribution, and reproduction in any medium for non commercial use (NonCommercial, or CC-BY-NC) provided the original author and source are credited. 
of data collected provides valid information on causality association, preventability and management of ADRs [3]. The objective of this observational cross-sectional study is to monitor and evaluate the ADR by the following steps:

- Identify the drugs causing frequent ADRs

- Identify the frequently affected organ system involved in the ADR

- Categorise the ADRs based on the severity.

\section{Methodology}

\section{Study design and Site}

This observational and cross-sectional study was conducted for 10 months from January 2019 to October 2019 in an inpatient setting of a tertiary care hospital in Mangalore.

\section{Study tools}

Adverse drug reaction reporting form

\section{Inclusion criteria}

Patients of all age groups,either sex, only in wards and critical units were included.

\section{Exclusion criteria}

Known allergies or previous history given by patients regarding drug allergies are excluded from this study. OPD patients are also excluded. The use of alternative system of medicines such as Ayurveda, Homeopathy, and Unani etc as well as over dosage, excess consumption, was excluded. Patients who are mentally retarded, drug addicted, suicidal tendencies or consumption of a drug in the influence of alcohol was also excluded.

\section{RESULTS AND DiSCUSSION}

Pharmacovigilance is the program conducted worldwide to report various adverse reactions occurring due to drugs that are already being marketed. The most common clinical presentation involved skin disorders. The common causal drug group was antibiotics followed by GI agents administered by intravenous route.

In our study both the genders were equally affected from ADR's. The ADR's were reported by filling the ADR reporting form and was analysed. A total of 43 ADR's were reported during the study period. Among them 22 patients (51\%) were females while 21 patients $(48 \%)$ were males. A similar study done by Doshi et al. stated that incidence of ADR is equal in both genders [4].

In a study done by Lian-qiu Min from 85 cases of ADR, 73 cases were caused by intravenous administration, indicating that ADR caused by intravenous administration route is much higher than other routes. Our study also indicates that intravenous route were the highest occurring comprising of $60.46 \%$ followed by $29.53 \%$ causing by oral route of administration[5]. This was because the drug administration route of intravenous infusion is taken as the main treatment method in inpatients. At intravenous infusion, drugs directly enter human circulation, with fast action and no first-pass effect, the pharmacological effects and adverse reactions are stronger than oral administration, and many factors induce $\mathrm{ADR}$, including $\mathrm{pH}$ value, osmotic pressure, temperature, particulates etc. The requirements of intravenous administration are much higher than other preparations, so improper preparation operation, low preparation quality, inappropriate drug combination, long-term preparation liquid placing, high impurity content and fast (low) infusion will cause ADR.

Prakash .H. Bhabhor et al. did a study which reported that the most commonly implicated organ system was skin and appendages (52.25\%) the major causative drug classes were antimicrobials (40.28\%) followed by central nervous system (23.61\%)[6]. In our study among the organ systems involved, skin membrane were mostly affected which consisted of $60.46 \%$ of ADR's followed by $13.95 \%$ of ADR's were present as CNS and neurological disorders. Gastrointestinal ADR's and Physiological disorders were seen in $11.62 \%$ and $6.97 \%$ of patients respectively. ADR's like cough and breathlessness were seen in $4.65 \%$ of patients. (Table 1) The most common ADR was Itching which may have caused due to drug induced histamine release by mast cells unrelated to the immune system or may have reflected a bona-fide drug related allergic reaction. 
Table-1: Organ system involved in ADR

\begin{tabular}{|l|l|l|}
\hline Organ system involved & No of adr & Percentage \\
\hline Subcutaneous disorders & 26 & $60.46 \%$ \\
\hline CNS and neurological disorders & 6 & $13.95 \%$ \\
\hline Gastro intestinal disorders & 5 & $11.62 \%$ \\
\hline Physiological disorders & 3 & $6.97 \%$ \\
\hline Respiratory and thoracic disorders & 2 & $4.65 \%$ \\
\hline Renal and Urinary disorder & 1 & $2.32 \%$ \\
\hline
\end{tabular}

In a study conducted by Sakuma et al. showed that antibiotics were most frequently associated with ADEs [7]. Similarly the largest numbers of reports in our study was associated with antibiotics. Most of the drug categories suspected to cause ADRs was related to antibiotics (39.53\%), G1 drugs (16.27\%). The anti-diabetics (13.95\%), anticoagulants and antihypertensives (9.30\%), opioids (4.65\%) and NSAID's (6.97\%) also have roles in ADRs (Figure1).

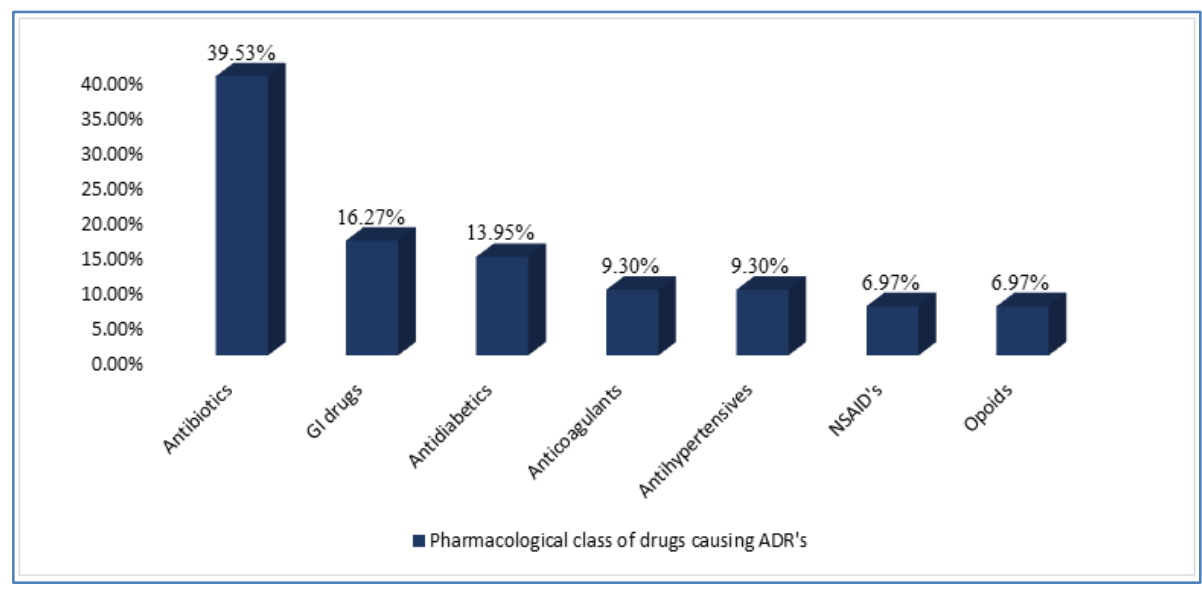

Fig-1: Pharmacological classes of drug implicated to cause adverse drug reaction

The most common generic drug producing ADR was Pantoprazole (13.95\%), Vancomycin (11.62\%) and Human Albumin (6.97\%). While drugs like Bisoprolol, Dexamethasone and Alprazolam showed fewer side effects (Figure 2).

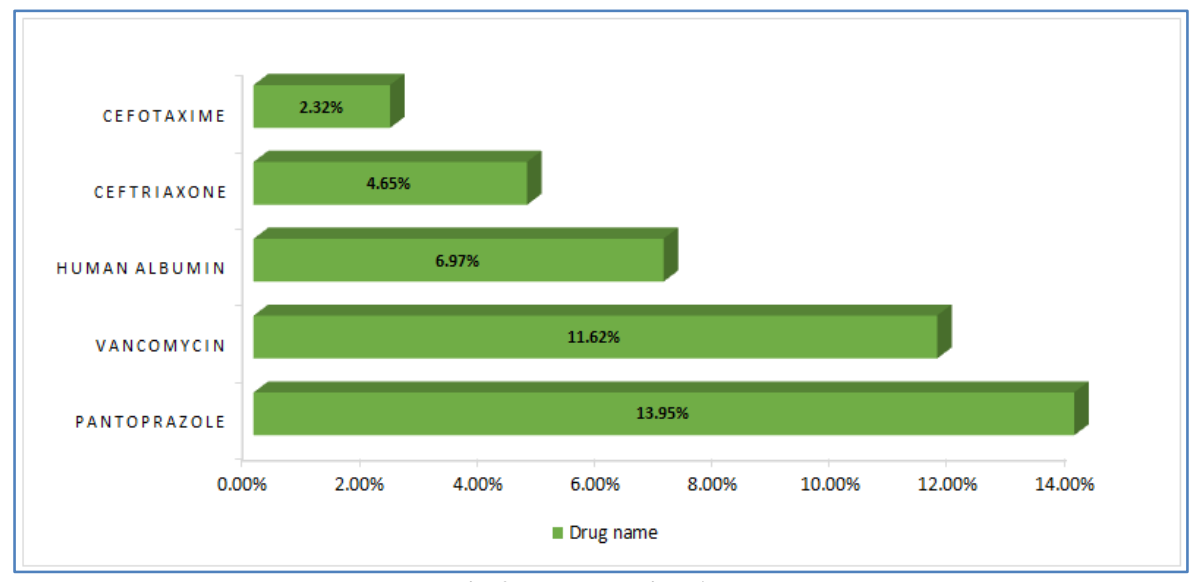

Fig-2: Drug causing ADR

Our study showed that majority of the ADR's $25(58 \%)$ were moderate, $11(26 \%)$ ADR's were found to be mild and $7(16 \%)$ of ADR's were found to be severe according to severity assessment. A study done by Shamna et al. that of reported ADR's moderate reactions accounted for 31(63.26\%) followed by mild reactions $14(28.57 \%)$. Only 4(8.16\%) of the reactions were severe [8] (Figure 3). 


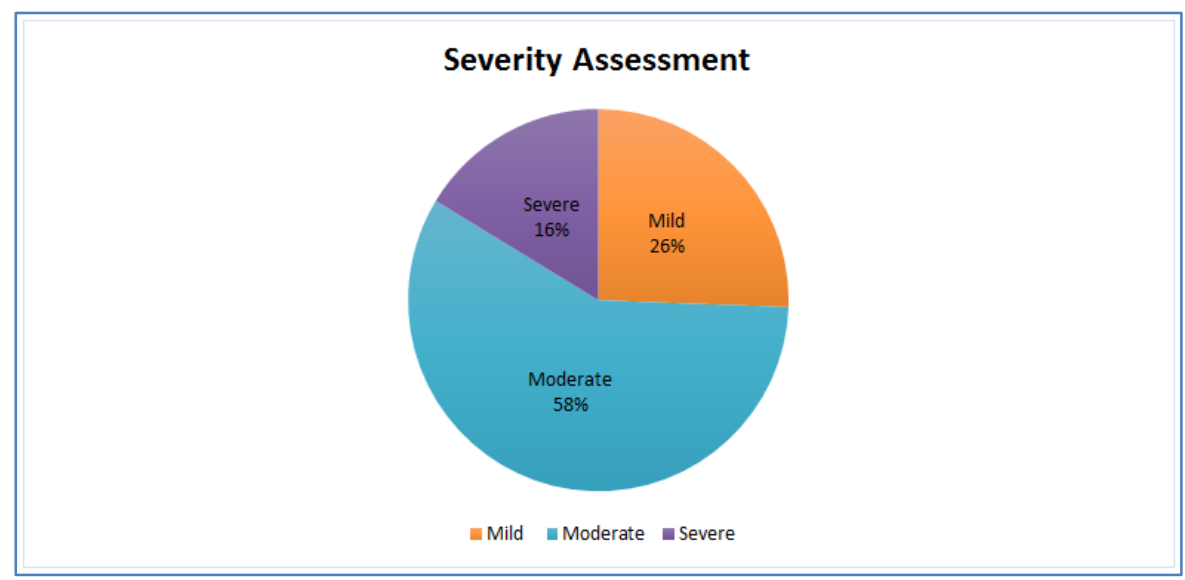

Fig-3: Severity assessment of ADR's by Hartwig and Siegal scale

\section{CONCLUSION}

Thus it can be concluded that adverse drug reaction is a significant limitation to the success of therapeutics. In order to deal with this problem Pharmacovigilance program is initiated. It is essential to improve the quality and quantity of ADR reports and to promote surveillance programs in health care facilities. It is worth providing some recommendations to improve the pharmacovigilance activities:

- A course in pharmacovigilance should be incorporated in the pharmacy and medicine curriculum, creating a culture of safety among students in the health care professions.

- Pharmacovigilance workshops and seminars should be conducted to provide guidance to healthcare providers for recognizing and reporting ADRs. Additionally, pharmacovigilance studies should be supported.

- ADR reporting by healthcare professionals and manufacturing companies as well as by patients and the public should be encouraged.

- Incentives should be provided to healthcare professionals reporting ADRs not associated with human errors.

- ADR forms should be collected periodically from health facilities by sending representatives.

\section{REFERENCE}

1. Mammen, S. J. (2018). A Study of Adverse Drug Reactions in a Tertiary care Hospital of Pune. PharmaTutor, 6(8), 38-43.

2. World Health Organization. (2002). Safety of medicines: A guide to detecting and reporting adverse drug reactions: Why health professionals need to take action (No. WHO/EDM/QSM/2002.2). World Health Organization.

3. Ramakrishnaiah, H., Krishnaiah, V., Pundarikaksha, H. P., \& Ramakrishna, V. (2015). A prospective study on adverse drug reactions in outpatients and inpatients of medicine department in a tertiary care hospital. Int $J$ Basic Clin Pharmacol, 4(3), 515-521.

4. Doshi, M. S., Patel, P. P., Shah, S. P., \& Dikshit, R. K. (2012). Intensive monitoring of adverse drug reactions in hospitalized patients of two medical units at a tertiary care teaching hospital. Journal of pharmacology \& pharmacotherapeutics, 3(4), 308.

5. Jin, X. Q., \& Min, L. Q. (2013). Analysis on 85 case reports of adverse drug reactions. African Journal of Traditional, Complementary and Alternative Medicines, 10(3), 508-515.

6. Bhabhor, P. H., Patel, T. K., Vahora, R., Patel, P. B., \& Desai, N. (2014). Adverse drug reactions in a tertiary care teaching hospital in India: analysis of spontaneously reported cases. Int J Basic Clin Pharmacol, 3(6), 1078-85.

7. Sakuma, M., Kanemoto, Y., Furuse, A., Bates, D. W., \& Morimoto, T. (2019). Frequency and severity of adverse drug events by medication classes: the JADE Study. Journal of patient safety.

8. Shamna, M., Dilip, C., Ajmal, M., Mohan, P. L., Shinu, C., Jafer, C. P., \& Mohammed, Y. (2014). A prospective study on Adverse Drug Reactions of antibiotics in a tertiary care hospital. Saudi pharmaceutical journal, 22(4), 303308.

9. S Rehan, H., Chopra, D., K Sah, R., \& Mishra, R. (2012). Adverse drug reactions: trends in a tertiary care hospital. Current drug safety, 7(5), 384-388.

10. Adhikari, A., Bhattacharjee, N., Bhattacharya, S., Indu, R., \& Ray, M. (2017). Evaluation of Adverse Drug Reactions in Tertiary Care Hospital of Kolkata, West Bengal, India. Journal of Young Pharmacists, 9(3), 311.

11. Prajapati, K., Desai, M., Shah, S., Panchal, J., Kapadia, J., \& Dikshit, R. (2016). An analysis of serious adverse drug reactions at a tertiary care teaching hospital. Perspectives in clinical research, 7(4), 181.

12. Lihite, R. J., Lahkar, M., Das, S., Hazarika, D., Kotni, M., Maqbool, M., \& Phukan, S. (2017). A study on adverse drug reactions in a tertiary care hospital of Northeast India. Alexandria journal of medicine, 53(2), 151-156. 
13. Malathi, M., Shanthi, M., \& Nambi, T. (2017). Adverse drug reactions at a tertiary care hospital in south india-A prospective analysis.

14. Geer, M. I., Koul, P. A., Tanki, S. A., \& Shah, M. Y. (2016). Frequency, types, severity, preventability and costs of adverse drug reactions at a tertiary care hospital. Journal of pharmacological and toxicological methods, 81, 323334.

15. Roy, K., Nadig, P., \& Prakash, B. (2015). Monitoring and analysis of adverse drugs reactions in a private tertiary care teaching hospital. Asian J Pharm Clin Res, 8(2), 335-7.

16. Kumar, B. N., Nayak, K., Singh, H., Dulhani, N., Singh, P., \& Tewari, P. (2010). A pharmacovigilance study in medicine department of tertiary care hospital in Chhattisgarh (Jagdalpur), India. Journal of young pharmacists, 2(1), 95-100.

17. Padmaja, U., Adhikari, P., \& Pereira, P. (2009). A prospective analysis of adverse drug reactions in a South Indian Hospital. Online Journal of Health and Allied Sciences, 8(3).

18. Rehan, H. S., Chopra, D., \& Kakkar, A. K. (2009). Physician's guide to pharmacovigilance: terminology and causality assessment. European journal of internal medicine, 20(1), 3-8.

19. Raut, A., Diwan, A., Patel, C., Patel, P., \& Pawar, A. (2011). Incidence, severity and financial burden associated with adverse drug reactions in medicine inpatients. Asian j pharm clin res, 4(2), 103-111.

20. Sriram, S., Ghasemi, A., Ramasamy, R., Devi, M., Balasubramanian, R., Ravi, T. K., \& Sabzghabaee, A. M. (2011). Prevalence of adverse drug reactions at a private tertiary care hospital in south India. Journal of research in medical sciences: the official journal of Isfahan University of Medical Sciences, 16(1), 16.

21. Pirmohamed, M., James, S., Meakin, S., Green, C., Scott, A. K., Walley, T. J., ... \& Breckenridge, A. M. (2004). Adverse drug reactions as cause of admission to hospital: prospective analysis of 18820 patients. Bmj, 329(7456), 15-19.

22. The pharmacovigilance news - National Drug Authority. Available at www.nda.or.ug/docs/bulletin\%20issue\% 20No.1.pdf. Last Accessed 29.10.2012.

23. Palaniappan, M., Sandhiya Selvarajan, M. G., Subramaniyan, G., Dkhar, S. A., Pillai, A. A., Jayaraman, B., \& Chandrasekaran, A. (2015). Pattern of adverse drug reactions reported with cardiovascular drugs in a tertiary care teaching hospital. Journal of clinical and diagnostic research: JCDR, 9(11), FC01.

24. Prajapati, K., Desai, M., Shah, S., Panchal, J., Kapadia, J., \& Dikshit, R. (2016). An analysis of serious adverse drug reactions at a tertiary care teaching hospital. Perspectives in clinical research, 7(4), 181.

25. Shamna, M., Dilip, C., Ajmal, M., Mohan, P. L., Shinu, C., Jafer, C. P., \& Mohammed, Y. (2014). A prospective study on Adverse Drug Reactions of antibiotics in a tertiary care hospital. Saudi pharmaceutical journal, 22(4), 303308. 